\title{
La fisioterapia y la cirugía obtienen similares resultados en el tratamiento de pacientes con rotura meniscal y artrosis
}

Physical Therapy and Surgery have similar results in the treatment of patients with a meniscal tear and osteoarthritis

Katz J.N. y col. N Engl J Med 2013;368(18):1675-84.

\section{Objetivos}

Evaluar la eficacia de la meniscectomía parcial artroscópica comparada con la fisioterapia estandarizada para el tratamiento de la rotura meniscal con artrosis leve a moderada concomitante.

\section{Diseño, lugar y pacientes}

Ensayo clínico multicéntrico (siete centros de EE.UU.), abierto, aleatorizado y controlado. Seguimiento final a un año. La aleatorización fue estratificada por sexo y grado de artrosis y en bloque, mediante el uso de un programa basado en la web. La asignación fue informada a los pacientes y al cirujano para comenzar el tratamiento entre las dos y cuatro semanas luego de la aleatorización.

De un total de 330 pacientes, 161 (71 hombres) de $59 \pm 8$ años fueron asignados a meniscectomía parcial artroscópica y 169 (71 hombres) de 57,8 \pm 7 años a fisioterapia.

\section{Medición de resultados principales}

El resultado primario fue cambios, respecto al basal, en la escala físico-funcional de WOMAC (Western Ontario and McMaster Universities Arthritis Index) a los seis meses postoperatorios.

Se estableció como diferencia mínima clínicamente significativa, 10 puntos o más, en la mejoría de la escala de WOMAC. El análisis fue por de intención de tratar modificado.

\section{Resultados principales}

Ambas intervenciones mejoraron la escala WOMAC a los 6 y 12 meses respecto al basal pero sin diferencias importantes entre ambas (Tabla 1). A los 6 meses 51 pacientes que habían sido asignados al grupo fisioterapia (30\%) fueron operados y 9 pacientes asignados a cirugía, no se habían operado (entrecruzamiento). No hubo diferencia en la frecuencia de efectos adversos entre los grupos.

Tabla 1. Resultados primarios y secundarios

\begin{tabular}{|c|c|c|c|}
\hline \multirow[b]{2}{*}{ Resultados principales } & \multicolumn{2}{|c|}{ Mejoria desde línea de base } & \multirow[b]{2}{*}{ Diferencia entre grupos } \\
\hline & $\begin{array}{c}\text { Artroscopia } \\
(\mathrm{N}=161)\end{array}$ & $\begin{array}{c}\text { Fisioterapia } \\
(\mathrm{N}=169)\end{array}$ & \\
\hline WOMAC a 6 meses (IC95\%) & $20,9(17,9$ a 23,9$)$ & $18,5(15,6$ a 21,5$)$ & $2,4(-1,8$ a 6,5$)$ \\
\hline WOMAC a 12 meses (IC95\%) & $23,5(20,5$ a 26,5$)$ & $22,8(19,8$ a 25,8$)$ & $0,7(-3,5$ a 4,9$)$ \\
\hline
\end{tabular}

\section{Conclusiones}

No se encontraron diferencias ni clínica ni estadísticamente significativas entre los grupos luego de seis meses de la aleatorización. Sin embargo el $30 \%$ de los pacientes asignados solamente a fisioterapia fueron operados dentro de los seis meses. El $70 \%$ restante de los asignados a fisioterapia no necesitó una cirugía, lo que avalaría el manejo inicial no quirúrgico de estos pacientes.

Fuente de financiamiento: National Institute of Arthritis and Musculoskeletal and Skin Diseases of the National Institutes of Health.

\section{Comentario}

En este ensayo clínico multicéntrico, que involucró a pacientes con rotura meniscal y evidencia imagenológica de artrosis de rodilla, no hubo diferencias significativas en la magnitud de mejoría funcional y de dolor a los 6 y 12 meses entre las ramas de tratamiento. Ensayos aleatorizados más pequeños mostraron que estos resultados persisten hasta los cinco años ${ }^{1,2}$.

Debe mencionarse que sólo se enrolaron un $26 \%$ de los pacientes elegibles, lo cual limita la validez externa. La razón más frecuente de no enrolamiento fue la preferencia por parte del paciente o del cirujano de un tratamiento sobre el otro (sesgo de selección). El ensayo no fue ciego ya que no fue factible de realizar un grupo de comparación simulado. A pesar de estas limitaciones, los resultados de este ensayo pueden ayudar en el manejo del cuidado de los pacientes con síntomas en la rodilla, ruptura meniscal y evidencia de artrosis en las imágenes. Por otro lado, se han reportado mayores índices de complicaciones y progresión de la artrosis luego de una meniscectomía artroscópica en pacientes con degeneración articular ${ }^{35}$.

\section{Conclusiones del comentador}

Dado que no hubo diferencias significativas en los resultados entre los grupos y que gran parte de los pacientes que realizaron fisioterapia no necesitaron operarse, existe justificación para el manejo inicial no quirúrgico de los pacientes con artrosis y lesiones meniscales, esperándose una mejoría del estado funcional y del dolor de la rodilla dentro de un periodo de 6 a 12 meses. La artroscopía correctamente indicada, ya sea por el fracaso del tratamiento conservador o por la prevalencia franca de síntomas mecánicos, como bloqueo por un fragmento meniscal desplazado, sigue teniendo su lugar, siendo costo-efectiva y con mejoría de la calidad de vida de los pacientes $^{6}$

Carlos Yacuzzi [ Servicio de ortopedia y traumatología del Hospital Italiano de Buenos Aires. carlos.yacuzzi@ hiba.org.ar ]

Yacuzzi C. La fisioterapia y la cirugía obtienen similares resultados en el tratamiento de pacientes con rotura meniscal y artrosis. Evid Act Pract Ambul. 2014:17(4).Oct-Dic. 125. Comentado de: Katz JN, Brophy RH, Chaisson CE, et al. Surgery versus physical therapy for a meniscal tear and osteoarthritis. N Engl J Med. May 2 2013;368(18):1675-84. PMID: 23506518.

\section{Referencias}

1. Herrlin $S$ y col. Arthroscopic or conservative treatment of degenerative medial meniscal tears: a prospective ran- domised trial. Knee Surg Sports Trauma- tol Arthrosc 2007:15:393-401.

2. Herrlin SV y col. Is arthroscopic surgery beneficial in treating non-traumatic, degenerative me- dial meniscal tears? A five year follow-up. Knee Surg Sports Traumatol Arthrosc 2013;21:358-64.

3. Muscolo DL1 y col. Osteonecrosis of the knee following arthroscopic meniscectomy in patients over 50-years old. Arthroscopy. 1996 Jun;12(3):273-9.

4. Englund M, Lohmander LS. Risk factors for symptomatic knee osteoarthritis fifteen to twenty-two years after meniscectomy. Arthritis Rheum. 2004;50:2811-9.

5. Mills PM y col. Tibiofemoral cartilage defects 3-5 years following arthroscopic partial medial meniscectomy. Osteoarthritis Cartilage. 2008;16:1526-31.

6. J. Lubowitz, D. Appely Cost-effectiveness analysis of the most common orthopaedic surgery procedures: Knee arthroscopy and knee anterior cruciate ligament reconstruction Arthroscopy 2011:1317-132. 